\title{
Hemmung der PCSK9 senkt VTE-Risiko
}

Marston NA et al. The Effect of PCSK9 (Proprotein Convertase Subtilisin/Kexin Type 9) Inhibition on the Risk of Venous Thromboembolism. Circulation 2020; 141: 1600-1607

Die Beziehung zwischen Cholesterinspiegel und dem Risiko für venöse Thromboembolien (VTE) ist bisher ungenügend verstanden. Observationsstudien haben unterschiedliche Ergebnisse berichtet - einige fanden einen Zusammenhang zwischen LDL-Spiegeln und einem erhöhten VTE-Risiko, andere konnten diese Assoziation nicht zeigen. Neuere genetische Studien haben jedoch einen möglichen Link aufgezeigt.

Die Autoren um Marston aus Boston untersuchten in ihrer Arbeit den Effekt einer Hemmung der Proprotein-ConvertaseSubtilisin/Kexin Typ 9 (PCSK 9) auf das VTE-Risiko; ferner untersuchten sie mögliche Mechanismen und die Effektivität der Inhibition in Subgruppen mit klinisch und genetisch definiertem Risiko. Dafür führten sie eine Post-hoc-Analyse des FOURIER-Trials durch, der untersucht hatte, ob Evolocumab das Risiko von VTE-Ereignissen reduziert. Ferner wurden Daten des FOURIER und ODYSSEY OUTCOMES in Metaanalysen untersucht, um den Klasseneffekt einer PCSK9-Hemmung auf das Risiko von VTE zu untersuchen. Außerdem untersuchten die Autoren Lipid-Ausgangswerte, um mögliche Mechanismen der VTE-Reduktion durch Evolocumab aufzuzeigen. Zudem wurde eine explorative genetische Analyse durchgeführt.

\section{Ergebnisse}

Im FOURIER lag die Hazard-Ratio (HR) für die VTE mit Evolocumab bei 0,71, es zeigte sich kein Effekt im ersten Jahr (HR: 0,96; 95\%-KI 0,57-1,62), jedoch eine Reduktion um $46 \%$ nach einem Jahr (HR: 0,54; $95 \%-K I$ $0,33-0,88 ; p=0,014)$. Die Metaanalyse des FOURIER und ODYSSEA OUTCOMES zeigte eine relative Risikoreduktion um $31 \%$ bei der VTE durch die Hemmung der PCSK9 (HR: 0,69; 95\%-KI 0,53-0,90; $\mathrm{p}=0$,007). Zwischen dem Ausgangswert des LDL und der Größe des VTE-Risikos gab es keine Beziehung. Patienten mit höheren Ausgangswerten des Lipoprotein-A-Levels hatten dagegen unter Evolocumab geringere Spiegel und ein geringes Risiko der VTE. Modelliert als kontinuierliche Variable zeigte sich eine signifikante Interaktion zwischen dem Lp(a)-Ausgangswert und der Größe der VTE-Risikoreduktion. Ein polygeneti- scher Risiko-Score identifizierte Patienten, die ein mehr als 2-fach erhöhtes Risiko für eine VTE hatten und die eine größere relative und absolute Risikoreduktion im Vergleich zu Patienten ohne hohes genetisches Risiko hatten.

\section{FAZIT}

Die Hemmung der PCSK9 reduzierte signifikant VTE-Ereignisse. Die Assoziation zwischen dem Grad der Lp(a)Senkung und der Größe der VTEReduktion legt die Vermutung nahe, dass Lp(a) ein Mediator dieses Effekts ist. Die Autoren schließen, dass dieser Befund aufgrund der aktuell laufenden Entwicklung von potenten LP(a)Inhibitoren von besonderem Interesse ist. Die Autoren nennen als eine wesentliche Limitation ihrer Studie unter anderem, dass die VTE kein vorher definierter Endpunkt des FOURIER-Trials und ihre Inzidenz gering war.

MOR Dr. med. Benedikt Lampl, Regensburg 